\title{
Design of High Power S-Band GaN MMIC Power Amplifiers for WiMAX Applications
}

\author{
$\underline{\ddot{O}_{m e r} \text { Cengiz }^{1}}, \ddot{O ̈ z g u ̈ r ~ K e l e k c ̧ i ~}^{1}$, Galip Orkun Arıcan ${ }^{1}$, Ekmel $\ddot{z z b a y}^{1}$, Osman Palamutçuoğullart ${ }^{2}$ \\ 1 Bilkent University, Nanotechnology Research Center,06800,Bilkent,Ankara,Turkey,omerc@bilkent.edu.tr \\ okelekci@bilkent.edu.tr,g_arican@bilkent.edu.tr,ozbay@bilkent.edu.tr
}

2 Istanbul Technical University, Electrical \& Electronics Faculty, 34469, Maslak, Istanbul, Turkey

opal@ehb.itu.edu.tr

\begin{abstract}
This paper reports two different $\mathrm{S}$ band GaN MMIC PA designs for WiMAX applications. First PA has a 42.6 dBm output power with a 55\%PAE @ $3.5 \mathrm{GHz}$ and $16 \mathrm{~dB}$ small signal gain in the 3.2-3.8 GHz frequency range. When two of these MMICs were combined by using off-chip Lange Couplers, $45.3 \mathrm{dBm}$ output power with a 45\%PAE @3.5 Ghz and $16 \mathrm{~dB}$ small signal gain were obtained with less than $0.2 \mathrm{~dB}$ gain ripple in the 3.3-3.8 GHz frequency range.
\end{abstract}

\section{Introduction}

As the market for wireless communicaton systems such as cellular, personal communications services, and worldwide interoperability for microwave access (WiMAX) coming closer to reality, radio frequency (RF) and microwave power amplifiers have attracted great attention [1-2]. The rapid development of the RF power electronics requires the introduction of wide bandgap material due to its potential in high output power density, high operation voltage and high input impedance [3]. A variety of power amplifier technologies are competing for market share, such as Si lateral-diffused metal-oxide-semiconductors (LDMOS) and bipolar transistors, GaAs metalsemiconductor field-effect transistors (MESFETs), GaAs (or GaAs/InGaP) heterojunction bipolar transistors, SiC MESFETs, and GaN high-electron mobility transistors (HEMTs) [4]. Compared to other compound III-V semiconductor materials, gallium nitride $(\mathrm{GaN})$ provides some superior material characteristics including wide bandgap, high breakdown field, high thermal conductivity, and high saturated velocity [2].

In this work two different GaN based MMIC designs for WiMAX applications will be presented. Agilent Advanced Design System (ADS) is used for design simulations and analyzes. MMICs were designed by using Cree GaN HEMT design kit named Cree_GaN_Design_Kit_v1.101a. Typical HEMT RF performances at $3.5 \mathrm{GHz}$ for this design kit are:

- $\quad 4.5 \mathrm{~W} / \mathrm{mm}$ power density

- $14 \mathrm{~dB}$ small signal gain

- High output impedance

- Low input capacitance

- $65 \%$ PAE

- 28 volt drain operation

Design goals are minimum $30 \mathrm{~W}$ output power, $15 \mathrm{~dB}$ small signal gain with gain ripple lower than $0.5 \mathrm{~dB}$ and maximum $-12 \mathrm{~dB}$ input return loss in the $3.3-3.7 \mathrm{Ghz}$ frequency range.

\section{3.5 GHz GaN MMIC Power Amplifier Design}

First GaN MMIC is designed with ideal lumped passive elements with $8 \times 500 \mu \mathrm{m}$ Cree transistor and second MMIC is its equivalent circuit with transmission lines and real passive elements.

$\Pi$ type resonance circuits used to match the input and output impedance of the $8 \times 500 \mu \mathrm{m}$ transistor to 50 ohm. Circuit schmatic can be seen in Figure 1. This kind of matching circuit gives the opportunity of bandwith flatness over the desired frequency range. 
$8 \times 500 \mu \mathrm{m}$ Cree transistor has a stability factor of $\mathrm{K} \leq 0.5$ at $3.5 \mathrm{Ghz}$ and below frequencies. Parallel R-C stability circuit is used at gate port of the transistor to achieve $\mathrm{K}>1$ at all frequencies to prevent any possible oscillation. The designed GaN MMIC circuit with ideal lumped elements and R-C stability circuit is shown in Figure 1. Biasing circuit is embedded into the input and output matching circuits which can be seen in Figure 1.

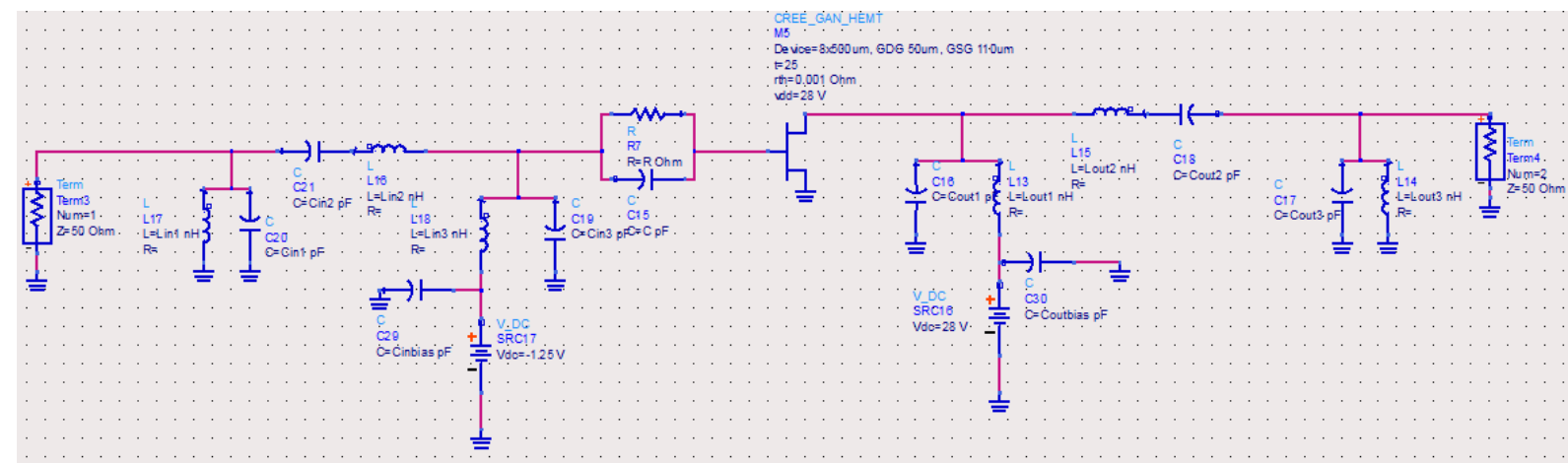

Figure 1: ADS schematic of the designed GaN MMIC Power Amplifier (PA) with ideal lumped elements

S parameter simulation results of the designed GaN MMIC are shown in Figure 2-a. The designed GaN MMIC has lower than $-14 \mathrm{~dB}$ input return loss at $3.5 \mathrm{GHz}$ and $16 \mathrm{~dB}$ small signal gain over the $3 \mathrm{GHz}-4 \mathrm{GHz}$ frequency band. It has a stability factor $\mathrm{K}>1$ over $0.5 \mathrm{GHz}-10 \mathrm{GHz}$ frequency band.

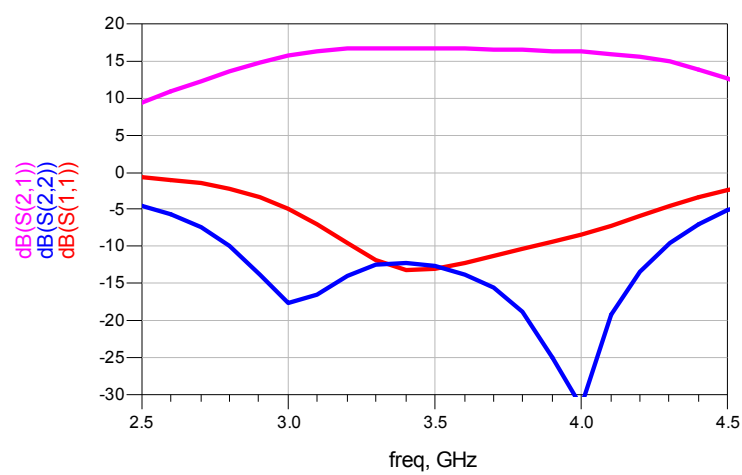

a)

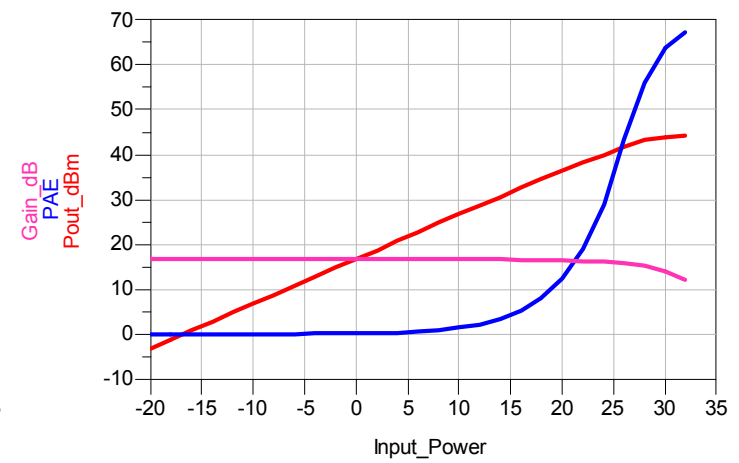

b)

Figure 2: a) S parameter simulation results b) Large signal simulation results, of the designed GaN MMIC PA with ideal lumped elements

Large signal simulation results are shown in Figure 2-b. According to the results GaN MMIC PA has a P1dB=42.2 $\mathrm{dBm}, \mathrm{P} 3 \mathrm{~dB}=43.9 \mathrm{dBm}$ and $65 \%$ PAE.

After obtaining the design goals with ideal lumped elements, the equivalent circuit with transmission lines and real passive elements is built. ADS layout of that MMIC is shown in Figure 3. 


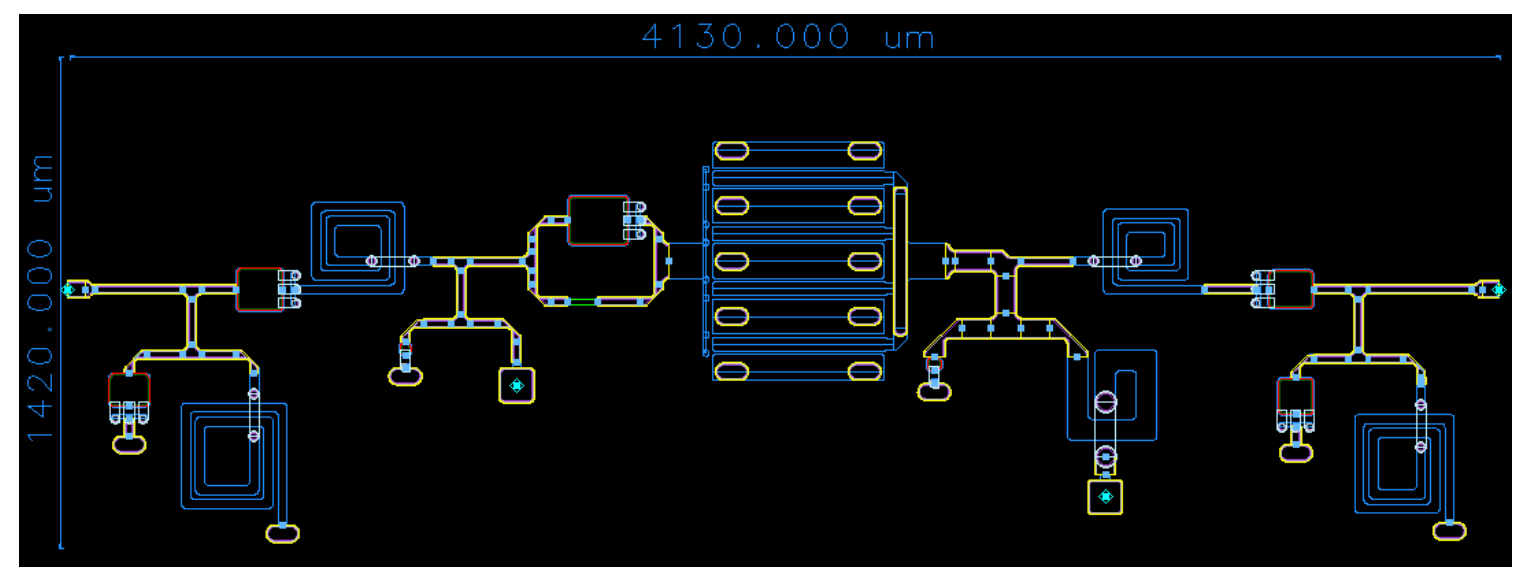

Figure 3: Circuit layout of the designed GaN MMIC PA

S parameter results can be seen in Figure 4-a. The designed GaN MMIC has 16 dB small signal gain over the 3.2 $3.8 \mathrm{GHz}$ frequency band and $-13.5 \mathrm{~dB}$ input return loss at $3.5 \mathrm{GHz}$.

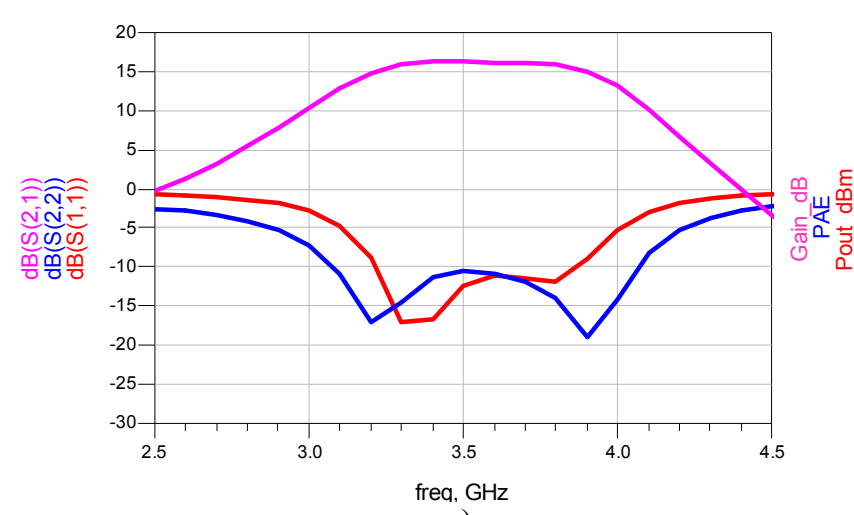

a)

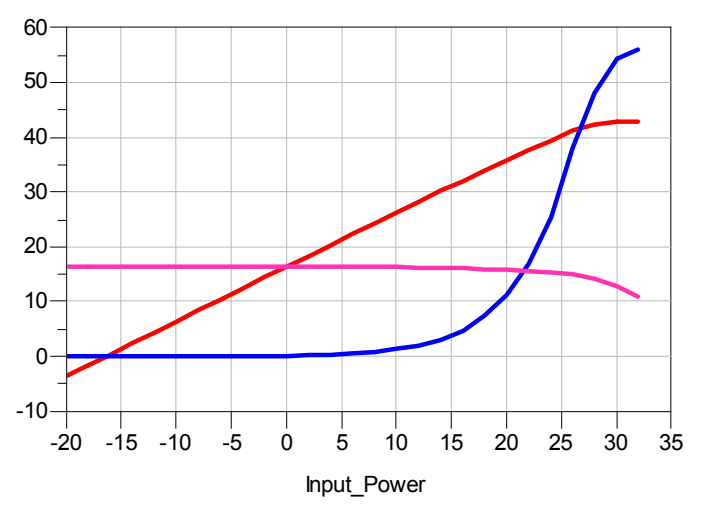

b)

Figure 4: a) S parameter simulation results b) Large signal simulation results, of the designed GaN MMIC PA

The designed GaN MMIC PA is unconditionally stable in $0.5 \mathrm{GHz}-10 \mathrm{GHz}$ frequency range. According to large signal simulation results as seen in Figure 4-b, the designed GaN MMIC has P1dB=41.6 dBm, P3dB=42.6 dBm and $55 \%$ PAE.

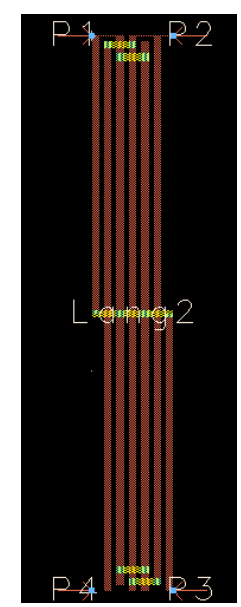

a)

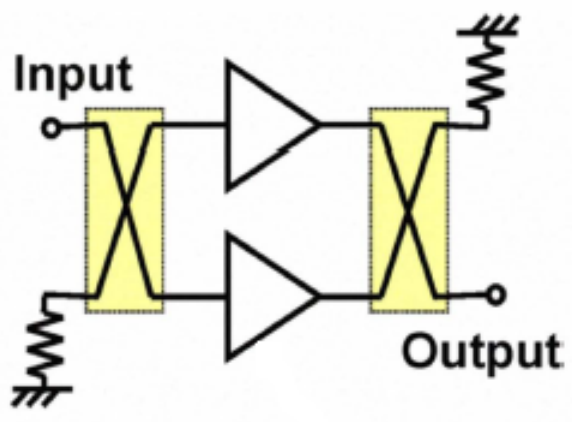

b)

Figure 5: a) Lange Coupler designed with Teflon PCB parameters @3.5 GHz b) Block diagram of the designed GaN MMIC PA 
We then combined two of the GaN MMICs with monolithic Lange couplers to increase its output power. Firstly, a coupler was designed to achieve the operation from 3.3 to $3.7 \mathrm{GHz}$ with Cree process parameters. According to design results, the designed coupler has a length of $8.9 \mathrm{~mm}$. Due to high cost of valuable Cree GaN wafer, it is not reasonable to manufacture such large area couplers as on-wafer. Therefore, it is decided to design and manufacture the couplers as off-chip. Then, Lange couplers were designed by using Teflon PCB parameters. Layout of Lange coupler which is designed by using Teflon PCB parameters is shown in Figure 5-a. A block diagram of the designed GaN MMIC PA is shown in Figure 5-b.

$\mathrm{S}$ parameter simulation results of the complete design can be seen in Figure 6-a. The designed GaN MMIC is unconditionally stable in the $0.5-10 \mathrm{GHz}$ frequency range. The designed GaN MMIC has $16 \mathrm{~dB}$ small signal gain over the 3.3 -3.8 GHz frequency band and lower than $-30 \mathrm{~dB}$ input return loss at $3.5 \mathrm{GHz}$.

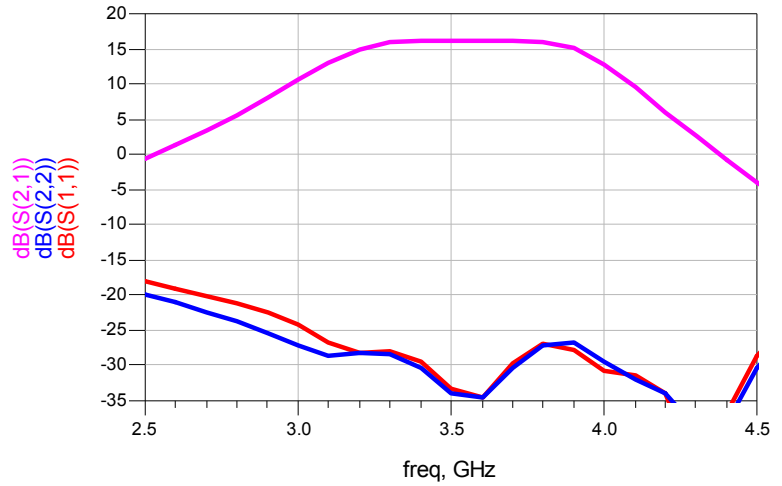

a)

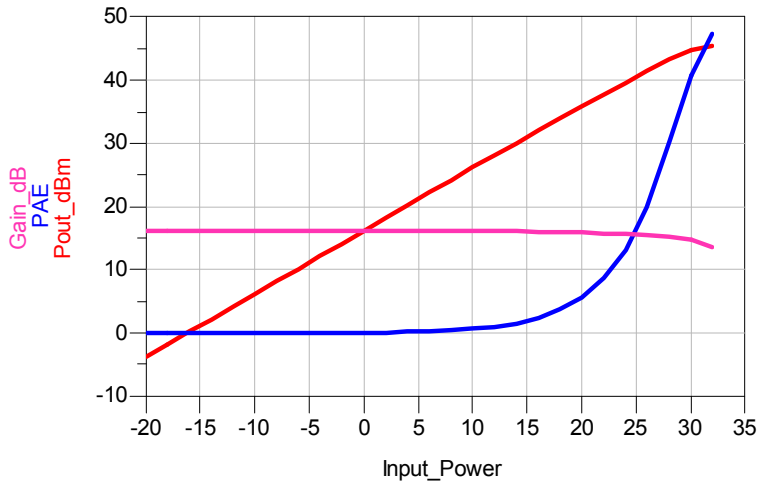

b)

Figure 6: a) S parameter simulation results b) Large signal simulation results, of the complete GaN MMIC PA with Lange couplers

The complete GaN MMIC PA has a $\mathrm{P} 1 \mathrm{~dB}=44.9 \mathrm{dBm}, \mathrm{P} 3 \mathrm{~dB}=45.3 \mathrm{dBm}$ and $45 \%$ PAE at 3.5 Ghz as seen at Figure 6b.

\section{Conclusion}

In this study, two $3.5 \mathrm{GHz}$ high power S-band GaN MMIC PA designs were presented. The first designed GaN MMIC PA shows an output power of $42.6 \mathrm{dBm}$ with 55\% PAE@3.5GHz and $16 \mathrm{~dB}$ small signal gain in the 3.2-3.8 GHz frequency range. In the second design, two of these GaN MMICs were combined with Lange Couplers in order to increase the output power of the PA. Due to high cost of valuable Cree GaN wafer, Lange couplers were designed and manufactured as off-chip by using Teflon PCB parameters. The second GaN MMIC PA exhibited 45.3 $\mathrm{dBm}$ output power with 45\% PAE @ $3.5 \mathrm{GHz}$ and $16 \mathrm{~dB}$ small signal gain with less than $0.2 \mathrm{~dB}$ gain ripple in the 3.33.8 GHz frequency range. Designed MMICs will be manufactured by using Shared Multi-Project Set (SMS) Mask Service of Cree GaN foundry. Based on these results, it can be concluded that GaN MMIC technology will take a big share from next generation WiMAX market.

\section{References}

1. U. K. Mishra, P. Parikh, and Y.-F. Wu, "AlGaN/GaN HEMTs-An overview of device operations and applications", Proceedings of the IEEE, vol. 90, no. 6, June 2002, pp. 1022-1031.

2. U.Schmid et al., "GaN devices for communication applications: evolution of amplifier architectures", International Journal of Microwave and Wireless Technologies, Cambridge University Press and the European Microwave Association, 2010, pp. 85-93.

3. U. K. Mishra, L. Shen, T. E. Kazior, and Y.-F. Wu, "GaN-Based RF Power Devices and Amplifiers", Proceedings of the IEEE, vol. 96, no. 2, February 2008, pp. 287 - 305.

4. S. Keller et al., "Gallium Nitride Based High Power Heterojunction Field Effect Transistors: Process Development and Present Status at UCSB”, IEEE Transactions on Circuits and Systems, vol. 48, no. 3, March 2001 , pp. 552 - 559 